\title{
Nature or nurture in finger counting: a review on the determinants of the direction of number-finger mapping
}

\section{Paola Previtali *, Luca Rinaldi and Luisa Girelli}

Department of Psychology, University of Milano-Bicocca, Milano, Italy

\section{Edited by:}

Liane Kaufmann, Private University for Health Sciences, Medical Informatics and Technology, Austria

\section{Reviewed by:}

Frank Domahs, RWTH Aachen University Hospital, Germany Judi Humberstone, University of Melbourne, Australia

\section{${ }^{*}$ Correspondence:}

Paola Previtali, Department of

Psychology, University of

Milano-Bicocca, Milano, Italy.

e-mail: p.previtali@campus.unimib.it
The spontaneous use of finger counting has been for long recognized as critical to the acquisition of number skills. Recently, the great interest on space-number associations shifted attention to the practice of finger counting itself, and specifically, to its spatial components. Besides general cross-cultural differences in mapping numbers onto fingers, contrasting results have been reported with regard to the directional features of this mapping. The key issue we address is to what extent directionality is culturally mediated, i.e., linked to the conventional reading-writing system direction, and/or biologically determined, i.e., linked to hand dominance. Although the preferred starting-hand for counting seems to depend on the surveyed population, even within the same population high inter-individual variability minimizes the role of cultural factors. Even if so far largely overlooked, handedness represents a sound candidate for shaping finger counting direction. Here we discuss adults and developmental evidence in support of this view and we reconsider the plausibility of multiple and coexistent number-space mapping in physical and representational space.

\section{COUNTING ON FINGERS TO COUNT}

The spontaneous use of fingers and other body parts to count and express numerosities has been reported since the pre-historic age (Ifrah, 1981) and appears to be almost universal, although highly variable across cultures. For example, for some tribes people (i.e., New Guineans), counting practice includes the whole body surface, as they orderly name and touch parts of the body starting with the little finger of the right-hand and ending with the left little finger, passing through the wrist, elbow, shoulder, eyes, nose, mouth, and ears (Ifrah, 1985), providing a track of the counted elements. With regard to the hands, while some ancient cultures, such as the Romans, used the left-hand alone to sign even large numerosities, e.g., 99 , in others, such as the Greeks, the right-hand was used as a counting tool (Lindemann et al., 2011). Interestingly, unimanual counting systems are still in use, mainly in Far-East cultures (e.g., Chinese, Japanese, and Korean Sign Language) although data on which of the two hands is used for counting are missing or mostly anecdotal.

Further evidence for the value of finger counting comes from its supportive role across development. The role of fingers in the development of numerical skills is well reflected by their massive use in the acquisition of simple arithmetic. Although the use of fingers mainly characterizes the initial stage of learning, this practice evolves with the increasing mastery of arithmetic knowledge (Jordan et al., 2008). Accordingly, indirect evidence for the role of fingers in supporting numerical development comes from studies reporting finger gnosis as a significant predictor of arithmetic performance in school-aged children (Fayol et al., 1998; Noël, 2005; Gracia-Bafalluy and Noël, 2008).

Recently, the long-lasting link between finger counting and number processing has received renewed attention within the embodied cognition approach, according to which cognitive processes are deeply shaped by the body's interaction with its environment (Wilson, 2002; Gibbs, 2006). In particular, numberto-finger associations have been shown to influence number processing (Sato et al., 2007; Domahs et al., 2011; Fischer and Brugger, 2011) and to modulate numerical mental representation (Di Luca et al., 2006; Fischer, 2008; Domahs et al., 2010). Specifically, there is evidence for an influence of finger counting direction on the direction of the mental numerical representation (Fischer, 2008) as well as for the specific structure of the finger counting system (e.g., the sub-base-five system) both on children's mental calculation (Domahs et al., 2008) and on adults' single-digit number comparison (Domahs et al., 2010). However, the functional relationship between fingers and number representation appears less obvious in specific sensory conditions. For example, it is worth noting that although blind children use their fingers in a less canonical way and less spontaneously than sighted children (Crollen et al., 2011), blind and sighted adults showed similar features in their mental representation of numbers (Castronovo and Seron, 2007; Sallilas et al., 2009). These results suggest that the contribution of finger counting to the mapping of numbers in the representational space may be less critical than considered thus far. Besides an increasing interest in finger counting practice, systematic investigations of its structural features, such as directionality, are still limited and mostly focused on cross-cultural differences (Lindemann et al., 2011). Yet, as with all motor actions, finger counting practice is expected to be modulated by hand preference, although it remains to be established to what extent handedness may further modulate abstract concepts (but see Casasanto, 2009; Casasanto and Chrysikou, 2011).

In this contribution we review the studies which have reported on finger counting practice disclosing the respective contributions of the cultural and biological determinants of its directionality 


\section{Box 1 | Key findings on finger counting direction.}

- Finger counting direction influences number processing (Di Luca et al., 2006; Sato et al., 2007).

- Finger counting direction shapes the mental numerical representation (Fischer, 2008; Domahs et al., 2010).
- The reading-writing system direction modulates finger counting direction (Lindemann et al., 2011).

- Different methods testing finger counting practice provide discrepant results (Sato and Lalain, 2008 vs. Fischer, 2008).
(Box 1). We first consider the evidence favoring the importance of cultural factors in shaping finger counting direction. Then, we turn our attention to the available data elucidating the influence of handedness in the adult population. Finally we will look to the recent developmental evidence that reflects the incremental influence of both biological and cultural factors in counting practice. We conclude that different space-number mappings (i.e., determined by cultural factors and/or finger counting habits) do coexist and distinctly emerge depending on the tasks' procedures and demands.

\section{DO CROSS-CULTURAL FACTORS DETERMINE DIFFERENT FINGER COUNTING DIRECTIONS?}

Although finger counting has been described in virtually all cultures (Ifrah, 1981), no universal counting routines have been observed, suggesting a great influence of cultural exposure in shaping the development of the finger counting practice (Pika et al., 2009). Indeed, different conventional patterns are used in finger-digit mapping, revealing a large cultural variability in the finger counting systems varying, for example, in dimensionality (i.e., base or sub-base systems), base size (i.e., 10 or 20 base), or extent (i.e., 27 or 30; Bender and Beller, 2011). This specificity has been observed also in counting direction asymmetries, as signed by the preferred starting-hand, which is the structural dimension we focus on. These cultural discrepancies are commonly attributed to the reverse orientation of the reading-writing system (i.e., leftto-right vs. right-to-left) that might induce a visuo-spatial asymmetry linked to the direction of scanning habits. In line with this interpretation, a large-scale online survey revealed a reversed preferential direction in Western (i.e., European and American) and Middle-Eastern (i.e., Iranian) populations. Indeed most Western participants $(68 \%)$ start counting preferentially on the left-hand, while Middle-Eastern individuals reported a reversed pattern with a preference to start with the right-hand (63\%; Lindemann et al., 2011). A further source of variability which emerged in this survey is related to the relative order of finger counting within a single hand. In particular, while Western populations reported to start counting on the thumb and to continue sequentially until the pinkie, Middle-Eastern individuals usually counted following the opposite order (i.e., from the pinkie to the thumb; Lindemann et al., 2011). Currently, the causal link between the relative direction in counting fingers within a hand (i.e., starting from the thumb or from the pinkie) and the starting-hand preference (i.e., starting from the left or right-hand) remains to be clarified due to the still limited evidence emerging from counting practice in Middle-Eastern populations. If the conventional scanning habit is a determinant of finger counting direction, intra-cultural differences should be minimal or absent. In contrast, this practice is not homogeneous even within the same Western sample, since the left-hand starting preference is marked in Anglo-Saxon countries (i.e., UK, USA, and Canada), but not in Belgians and Italians. This evidence partially confirms the role of cultural effects but minimizes the influence of the writing system direction in predicting starting preference.

For example, a large-scale questionnaire used to investigate finger counting patterns in a Scottish sample reported a preference (66\%) to start counting on the left-hand (Fischer, 2008). On the contrary, a direct test of hand preference in Italian (Di Luca et al., 2006; Sato et al., 2007) and French (Sato and Lalain, 2008) populations revealed that most individuals preferred to start counting on their right-hand ( $82 \%$ overall, 100 and $69 \%$ respectively). These contradictory results may well be attributed to the different methods adopted to assess finger counting, that is via written questionnaire or via direct observation. Aware of this difference, Lindemann et al. (2011) ran a control experiment comparing the two modalities within a group of participants on the basis of which they denied a modulation effect of the response mode. However, they considered only a homogeneous subgroup of individuals (English speakers of unknown handedness) different from those tested by enacting the finger count (Di Luca et al., 2006; Sato et al., 2007; Sato and Lalain, 2008). Yet, since the focus of our attention is a motor routine, a spontaneous and overlearned practice, the possible gap between enacting and reporting is expected to be significant. While the former procedural task involved an obvious implicit component, the latter requires explicit access to finger counting representations. For these reasons, we believe that task specific effects on finger counting deserve further attention in future research.

In conclusion, all data collected thus far clearly indicate that finger counting habits may vary substantially both within and between cultures, suggesting that reading-writing system direction is not the only factor that modulates the starting-hand preference during finger counting execution. Individual differences within the same population could be explained by taking handedness into consideration, since handedness indeed shapes many other motor activities.

\section{IS LEFT- AND RIGHT- STARTING A DOMINANCE MATTER?}

In principle, at least in Western cultures whose counting system involves two hands, finger counting practice is expected to be shaped, as any other bimanual action, by the lateral asymmetry determining hand dominance. For this reason, most studies adopt right-handedness as a recruiting criterion (Di Luca et al., 2006; Sato et al., 2007; Brozzoli et al., 2008) preventing any conclusive remarks on the role of hand dominance in finger counting direction. Indeed, when right-handed participants were recruited they showed a preference to start counting with their right-hand (Di Luca et al., 2006; Sato et al., 2007). Although a cultural effect might not be excluded (i.e., in both studies participants were Italians), homogeneous right-handedness may well be a confounding factor. 
Moreover, regardless of handedness, assessment of finger counting direction has thus far not been systematic even in studies focused on embodied numerosities (Domahs et al., 2010).

The first study to directly investigate the link between handedness and direction of finger counting in adulthood did not find any association between the two (Fischer, 2008); the proportion of the left- and right-hand starters (i.e., participants who started to count with the left- and the right-hand respectively) was the same among left- (70 and 30\% respectively) and right- handed (66 and 34\% respectively) Scottish individuals. This pattern would suggest that finger counting direction is unrelated to hand preference ( $p>0.05)$, although in this case the testing modality, i.e., written questionnaire, might have favored a left-to-right mapping, consistent with the Scottish reading habit direction.

In contrast, a more pronounced left-starting preference among left-handers has also been observed (Sato and Lalain, 2008; Previtali and Girelli, 2009; Lindemann et al., 2011). In particular, when finger counting was directly observed during its execution, a significant interaction between number-finger mapping and hand dominance was found. That is, French participants who started to count with their right-hand showed higher right-hand preference in unimanual activities (Sato and Lalain, 2008). It worth noting that in this study only three left-handers were tested but, despite this highly unbalanced sample (i.e., 3 vs. 97), the left-handed individuals consistently started to count with the left-hand.

A larger sample of left-handers was recently evaluated through an online survey (Lindemann et al., 2011), but in this case handedness was further qualified by cross-cultural differences. Indeed, the authors reported a more pronounced left-starting preference among Western left-handers $(36 / 40, p<0.01)$ but not within Middle-Eastern left-handers $(p>0.1)$ reflecting the interplay of both biological and cultural determinants in modeling finger counting practice.

However, a further contribution on a considerable number of left-handed $(N=30)$ and right-handed $(N=57)$ Italian participants reports a highly significant correlation between handedness and finger counting direction. An assessment of handedness by the Edinburgh Handedness Inventory (Oldfield, 1971) and a direct observation of finger counting revealed that $83 \%$ of left-handers were left-starters and $86 \%$ of right-handers were right-starters (Previtali and Girelli, 2009).

This effect of laterality also emerged in deaf signers who, like hearing speakers, exhibit a prevalence of right-hand dominance with the relative preference to sign numbers up to five with the right-hand and, for two-handed numbers (6-10), to sign the five-hand shape with the left-hand (Iversen et al., 2006). Similarly, a recent study on blind and sighted children (Crollen et al., 2011) showed that while sighted participants started to count with the dominant hand, i.e., $92 \%(N=11 / 12)$ of the right-handed children started counting with their right-hand and the only lefthanded child started counting with his/her left-hand, in blind participants the modulation of hand dominance was less systematic [ $54 \%,(N=6 / 11)$ of the right-handers were right-starters, the only left-hander was also a left-starter]. Whether the reduced laterality effect in blind participants is due to their less systematic use of finger counting and/or to the role of sighting in typical cerebral lateralization or handedness (Caliskan and Dane, 2009) remains to be established. Although the low proportion of lefthanders in the general population is a critical drawback that necessitates further cumulative data, handedness appears, so far, to be an effective predictor of the structural components of finger counting routines. Thus, as with any other bimanual action, the use of fingers in counting practice is intrinsically related to hand dominance.

\section{LOOKING BACK FOR EARLY INDEXES OF DIRECTIONALITY: HOW DO CHILDREN COUNT?}

Developmental data may provide a critical argument to the nature-nurture debate on the origin of the finger counting practice. Indeed, fingers are spontaneously used very early in development, well before the acquisition of reading and writing abilities (Fuson, 1988; Noël, 2005) and even occasionally preceed verbal labels in counting practice (Brissaud, 1992), although the influence of biological and cultural factors both increase incrementally over time. On the one hand, the influence of the dominant scanning direction associated with the writing system has been shown to emerge early in development, contributing to the occurrence of visuo-spatial asymmetries (Fagard and Dahmen, 2003; Opfer et al., 2010). On the other hand, although the first signs of lateral asymmetries emerge very early, handedness develops slowly, influenced by both genetic and cultural factors (Fagard and Dahmen, 2004), and increases in consistency during childhood (Mc Manus et al., 1988).

Importantly, the only study that has effectively examined potential differences across development (Sato and Lalain, 2008) suggests a stability of finger counting direction from childhood to adulthood. In fact, four different age groups ( $4-5$ years old, $6-$ 7 years old, 10-11 years old, and 24-47 years old) of French individuals evaluated in a finger counting task revealed, irrespectively of age, the same pattern in finger-number mapping (i.e., the righthand used to count from one to five and the left-hand used to count from six to nine).

In contrast, in a large Finnish group, right-handers were mostly right-starters $(60 \%)$ across ages, while a shift in finger counting direction occurred for left-handers, i.e., $100 \%$ of left-handed preschoolers were left-starters while only $50 \%$ of left-handed fourth graders started to count with their left-hand. This evidence supports the hypothesis that cultural factors modulate counting routines to some extent, although starting to count on the dominant hand is more frequent in both left- and right-handed participants of all ages (Räsänen and Koponen, 2010).

Finally, a recent study by Rinaldi and Girelli (2011) investigated the development of number-space associations in both the extra-personal physical space (e.g., counting visual arrays of elements), and in the personal space (i.e., finger counting), in 90 Italian-speaking 3- to 6- year-old preschoolers. Finger-digit mapping was assessed by spontaneous finger counting (from 1 to 10) and by requiring number-finger configurations (montring task, e.g., "Show me four with your fingers," $N=1-9$ ).

Seventy-three percent of the children started to count with their right-hand and $65 \%$ of children used the right-hand first to show numerosities, supporting the idea of a strong relationship between small digits and right-hand fingers and between large digits and left-hand fingers (Di Luca et al., 2006; Sato et al., 2007). In 
particular, the number of preschoolers who showed a stable association in both the counting and the montring tasks ( 32 children from right-to-left, 10 from left-to-right) increased with age and, interestingly, these children outperformed their peers in number comprehension. With regard to handedness, no systematic relation was found with the direction of finger counting (note that only five subjects were left-handers). Finally, the results revealed no stable relationship between the embodied, i.e., related to finger mapping, and disembodied, i.e., related to the spatial arrangement of the counted elements, mapping, suggesting flexible use depending on the context (Di Luca et al., 2006). Specifically, children counting right-to-left on their fingers (embodied mapping) point left-toright counting elements in the extra-personal space (disembodied mapping) and vice versa. In conclusion, this study may suggest that these two types of spatial mapping (i.e., embodied and disembodied) may differ, but coexist to support numerical comprehension from a very early age.

The relevance of finger counting in the acquisition and development of numerical skills has been long recognized, but only recently has this routine been linked to the way in which numbers are processed and mentally represented both throughout development and in adulthood (Di Luca et al., 2006; Sato et al., 2007; Brozzoli et al., 2008; Fischer, 2008; Sato and Lalain, 2008).

\section{REFERENCES}

Bender, A., and Beller, S. (2011). Fingers as a tool for counting - naturally fixed or culturally flexible? Front. Psychol. 2:256. doi:10.3389/fpsyg.2011.00256

Brissaud, R. (1992). "A tool for number construction: finger symbol sets," in Pathways to Number: Children's Developing Numerical Abilities, eds J. Bideaud, C. Meljac, and J.-P. Fisher (Hillsdale, NJ: Lawrence Erlbaum Associates), 41-67.

Brozzoli, C., Ishihara, M., Göbel, S. M., Salemme, R., Rossetti, Y., and Farne, A. (2008). Touch perception reveals the dominance of spatial over digital representation of numbers. Proc. Natl. Acad. Sci. U.S.A. 105, 5644-5648.

Caliskan, E., and Dane, S. (2009). Lefthandedness in blind and sighted children. Laterality 14, 205-213.

Casasanto, D. (2009). Embodiment of abstract concepts: good and bad in right- and left-handers. J. Exp. Psychol. Gen. 138, 351-367.

Casasanto, D., and Chrysikou, E. G. (2011). When left is "right": motor fluency shapes abstract concepts. Psychol. Sci. 22, 419-422.

Castronovo, J., and Seron, X. (2007). Semantic numerical representation in blind subjects: the role of vision in the spatial format of the mental number line. Q. J. Exp. Psychol. 60, 101-119.

Crollen, V., Mahe, R., Collignon, O., and Seron, X. (2011). The role of vision in the development of finger-number interactions: fingercounting and finger-monitoring in blind children. J. Exp. Child. Psychol. 109, 525-539.

Di Luca, S., Grana, A., Semenza, C., Seron, X., and Pesenti, M. (2006). Finger-digit compatibility in Arabic numeral processing. Q. J. Exp. Psychol. 59, 1648-1663.

Domahs, F., Klein, E., Moeller, K., Nuerk, H.-C., Yoon, B.-C., and Willmes, K. (2011). Multimodal semantic quantity representations: further evidence from Korean sign language. Front. Psychol. 2:256. doi:10.3389/fpsyg.2011.00256

Domahs, F., Krinzinger, H., and Willmes, K. (2008). Mind the gap between both hands: evidence for internal finger-based number representations in children's mental calculation. Cortex 44, 359-367.

Domahs, F., Moeller, K., Huber, S., Willmes, K., and Nuerk, H. C. (2010). Embodied numerosity: implicit hand-based representations influence symbolic number processing across cultures. Cognition 116, 251-266.

Fagard, J., and Dahmen, R. (2003). The effects of reading-writing direction on the asymmetry of space perception and directional tendencies: a comparison between French and Tunisian children. Laterality 8, 39-52.

Fagard, J., and Dahmen, R. (2004). Cultural influences on the development of lateral preferences: a

However, despite this renewed attention, detailed investigations on the structural features, i.e., directionality, of finger counting are still missing. Due to the differences observed in testing different populations, cultural determinants (i.e., the writing system direction) in shaping finger counting routines have been acknowledged (Lindemann et al., 2011). Nevertheless, when controlled for, the prediction that handedness represented a sound candidate for shaping finger counting direction was confirmed (Sato and Lalain, 2008; Previtali and Girelli, 2009). In conclusion, the interplay of cultural and biological determinants well account for both adults' (Lindemann et al., 2011) and children's (Räsänen and Koponen, 2010) counting practice, but further studies are needed to fully understand their relative role in shaping finger counting direction. In particular, within the embodied cognition approach, structural features of finger counting practice should be systematically assessed in order to significantly enlarge samples, especially those of left-handed participants. Finally, developmental and cross-cultural studies represent an ideal approach to disentangle the relative contribution of cultural, e.g., the reading-writing system direction, and biological, e.g., manual laterality, factors in shaping finger-number associations, especially due to their relevance in supporting number comprehension (Noël, 2005; Rinaldi and Girelli, 2011).

comparison between French and Tunisian children. Laterality 9 , 67-78.

Fayol, M., Barrouillet, P., and Marinthe, C. (1998). Predicting arithmetical achievement from neuropsychological performance: a longitudinal study. Cognition 68, B63-B70.

Fischer, M. H. (2008). Finger counting habits modulate spatialnumerical associations. Cortex 44 386-392.

Fischer, M. H., and Brugger, P. (2011). When digits help digits: spatial-numerical associations point to finger counting as prime example of embodied cognition. Front. Psychol. 2:260. doi: 10.3389/fpsyg.2011.00260

Fuson, K. C. (1988). Children's Counting and the Concepts of Number. New York: Springer.

Gibbs, R. W. (2006). Embodiment and Cognitive Science. Cambridge: University Press.

Gracia-Bafalluy, M., and Noël, M. P. (2008). Does finger training increase numerical performance? Cortex 44 , 368-375.

Ifrah, G. (1981). The Universal History of Numbers: From Prehistory to the Invention of the Computer. London: The Harvill Press.

Ifrah, G. (1985). From One to Zero: A Universal History of Numbers. New York: Viking Penguin.

Iversen, W., Nuerk, H., Jäger, L., and Willmes, K. (2006). The influence of an external symbol system on number parity representation, or what's odd about 6? Psychon. Bull. Rev. 13, 730-736.

Jordan, N. C., Kaplan, D., Ramineni, C., and Locuniak, M. N. (2008). Development of number combination in skill in the early school years: when do fingers help? Dev. Sci. 11, 662-668.

Lindemann, O., Alipour, A., and Fischer, M. H. (2011). Finger counting habits in middle-eastern and western individuals: an online survey. J. Cross Cult. Psychol. 42, 566-578.

Mc Manus, I. C., Sik, G., Cole, D. R., Mellon, A. F., Wong, J., and Kloss, J. (1988). The development of handedness in children. Br. J. Dev. Psychol. 6, 257-273.

Noël, M. P. (2005). Finger gnosia: a predictor of numerical abilities in children? Child Neuropsychol. 11, 413-430.

Oldfield, R. C. (1971). The assessment and analysis of handedness: the Edinburgh inventory. Neuropsychologia 9, 97-113.

Opfer, J. E., Thompson, C. A., and Furlong, E. E. (2010). Early development of spatial-numeric associations: evidence from spatial and quantitative performance of preschoolers. Dev. Sci. 13, 761-771.

Pika, S., Nicoladis, E., and Marentette, P. (2009). How to order a beer: cultural differences in the use of conventional gestures for numbers. J. Cross Cult. Psychol. 40, 70-80. 
Previtali, P., and Girelli, L. (2009). "Mapping numbers on the hand and on the head: which direction?" in Poster Presented at Workshop in Cultural Effects on the Mental NumberLine, York.

Räsänen, P., and Koponen, T. (2010). "Development of children's fingercounting habits," in Poster Presented at the Meeting in Honour of Professor Brian Butterworth: "Numbers in the Brain," London.

Rinaldi, L., and Girelli, L. (2011). "The role of number-space associations in preschoolers' numerical competence," in Poster Presented at the Workshop in Typical and Atypical
Neurocognitive Aspects of Numerical Processing, Beer Sheva.

Sallilas, E., Granà, A., El-Yagoubi, R., and Semenza, C. (2009). Numbers in the blind's "eye." PLOS ONE 4, e6357. doi:10.1371/journal.pone. 0006357

Sato, M., Cattaneo, L., Rizzolatti, G., and Gallese, V. (2007). Numbers within our hands: modulation of corticospinal excitability of hand muscles during numerical judgment. J. Cogn. Neurosci. 19, 684-693.

Sato, M., and Lalain, M. (2008). On the relationship between handedness and hand-digit mapping in finger counting. Cortex 44, 393-399.

Wilson, M. (2002). Six views of embodied cognition. Psychon. Bull. Rev. 9, 625-636.

Conflict of Interest Statement: The authors declare that the research was conducted in the absence of any commercial or financial relationships that could be construed as a potential conflict of interest.

Received: 01 August 2011; accepted: 18 November 2011; published online: 06 December 2011.
Citation: Previtali P, Rinaldi $L$ and Girelli L (2011) Nature or nurture in finger counting: a review on the determinants of the direction of number-finger mapping. Front. Psychology 2:363. doi: 10.3389/fpsyg.2011.00363

This article was submitted to Frontiers in Cognition, a specialty of Frontiers in Psychology.

Copyright (c) 2011 Previtali, Rinaldi and Girelli. This is an open-access article distributed under the terms of the Creative Commons Attribution Non Commercial License, which permits noncommercial use, distribution, and reproduction in other forums, provided the original authors and source are credited. 